University of Nebraska - Lincoln

DigitalCommons@University of Nebraska - Lincoln

Faculty Publications in Computer \& Electronics Electrical \& Computer Engineering, Department Engineering (to 2015)

2012

\title{
An Adaptive Algorithm for Corrupted Images
}

Deborah D. Duran-Herrmann

University of Nebraska-Lincoln

Qilin Qi

University of Nebraska-Lincoln

Yaoqing Lamar Yang

University of Nebraska-Lincoln, yyang3@unl.edu

Follow this and additional works at: https://digitalcommons.unl.edu/computerelectronicfacpub

Part of the Computer Engineering Commons

Duran-Herrmann, Deborah D.; Qi, Qilin; and Yang, Yaoqing Lamar, "An Adaptive Algorithm for Corrupted Images" (2012). Faculty Publications in Computer \& Electronics Engineering (to 2015). 75.

https://digitalcommons.unl.edu/computerelectronicfacpub/75

This Article is brought to you for free and open access by the Electrical \& Computer Engineering, Department of at DigitalCommons@University of Nebraska - Lincoln. It has been accepted for inclusion in Faculty Publications in Computer \& Electronics Engineering (to 2015) by an authorized administrator of DigitalCommons@University of Nebraska - Lincoln. 


\title{
An Adaptive Algorithm for Corrupted Images
}

\author{
Deborah D. Duran-Herrmann, Qilin Qi, and Yaoqing (Lamar) Yang \\ Department of Computer and Electronics Engineering \\ University Of Nebraska - Lincoln \\ Omaha, NE 68182, USA \\ Email: yyang3@unl.edu
}

\begin{abstract}
Noise is ever-present in communication channels and can produce devastating degradation to the images at the receiver end. In this paper, a novel approach to detecting different types of noise models in corrupted images is presented. Basically, it is a two-step process that has the ability to handle real-time applications due to its computational simplicity. The proposed algorithm handles error correction more efficiently in the second stage of processing, given a means to detect the type of noise present in the first stage. This algorithm will be verified and tested against existing methods on different noise models. Preliminary results show that this methodology can accurately detect and compensate for various noise sources common in multimedia applications, such as impulse, Gaussian, and uniform noise, with minimal loss to the integrity of the original digital image.
\end{abstract}

Keywords- Image Restoration, Corrupted Images, Mean Filters, Median Filters, Denoising, Noise Detection

\section{INTRODUCTION}

Noise can be unavoidable in communication networks, and its presence can have disastrous effects upon the data being sent. Since the introduction of the telephone and subsequent transmission of analog, then digital data, researchers have tried different techniques in order to either preserve the original transmission signal through use of amplifiers and repeaters with error correcting capabilities along the medium, or apply reconstructive techniques on the receiver end. The overall goal has always been to receive as accurate as possible what is being sent in spite of network conditions or noisy channels.

In multimedia applications, the process becomes more challenging. At transmission speeds that average 25-30 frames per second, the receiving end must be able to compensate by offering a real-time solutions for minimal distortion and packet loss. For a receiver with unlimited processing power, this still might not be an issue. However, the reality is that the receiver does have limited processing capability. Hence, the algorithms used to detect the presence of noise and eliminate it will have to be, theoretically, as computationally simple as possible. A well-defined process of detecting certain types of noise in transmitted images would have to be a somewhat crude for speed, yet reliable enough to accurately determine the type of filtering technique needed as images come in.

In this paper, we propose a new dynamic adaptive algorithm that will be able to allow the receiver to determine what filtering method is needed for the type of noise encountered. It is an extension of the methodology proposed in
[8] that can be modified to handle multimedia streams in realtime.

The remainder of this paper is organized as follows. In Section II, the related works on impulse noise detection and compensation are reviewed, and the basic difference of our work and contribution are highlighted. Section III describes the proposed system architecture for the adaptive algorithm and analyzes the methodology behind the adaptive algorithm for noise reduction in corrupted images. Section IV demonstrates the simulation results and the analysis of the experimental work utilizing the adaptive algorithm on a number of images and compares them to existing methods. Finally, future work and a summary of contributions are presented in Section V.

\section{RELATED WORK}

Previous work on methods for image noise compensation has been proposed in literature, e.g., [1] - [10]. Many of the current papers dealing with noise in communication networks propose a two-stage method of impulse noise reduction where noise is detected in the first stage and compensated for in the second using a filtering technique [4]. The first stage typically involves setting threshold parameters according to the statistical characteristics of the corrupted image and determining membership sets of pixels depending on detection of pixel corruption. Recursive methods have proven valuable in the filtering process by updating associating pixel membership sets for each iteration of the algorithm until an acceptable level of error is reached.

In Luo's papers [2], [3], and [7], a variety of efficient methods of impulse noise removal are introduced that utilized different detection schemes and noise removal processes. An alpha-trimmed mean filter is used in [2], improving on existing pixel value estimation approach similar to the detection process in [9]. Noisy pixels are replaced with a linear combination of the median of the local window surrounding the pixel and the original value of the pixel itself. In [3], a comparison is made between fuzzy impulse detection proposed and the performance of median filters. A unique histogram of the image is analyzed to determine if the pixel in question matches one of the peak locations in the histogram of the noisy image. The noisy pixel is "flagged" as belonging to the membership of noisy pixels. These noisy pixels are then replaced by a linear combination of the original value of the pixel and a median filter. The proposed method in [7] describes a three-step process: impulse noise detection, refinement, and noise cancellation. Again, a fuzzy technique is used to detect the noisy pixels while the refinement stage 
further defines the membership sets of noisy and non-noisy pixels. The method of noise cancellation is similar to [3].

Schulte, et.al. [1], [4], describe a fuzzy impulse noise detection and reduction method for both grayscale and color images. The nonlinear filtering technique used is accomplished in two stages: impulse noise detection and an edge-preserving reduction stage. For each pixel that is determined a noisy pixel, its corresponding grayscale value is stored in a histogram. Once the entire image is checked for the presence of noise, the fuzzy averaging of pixels in the immediate neighborhood of each determined noisy pixel is done to produce results with a low execution time. This method is extended to color images in [4].

Mathematical morphology is utilized in [5] in order to restore the pixels damaged by salt-and-pepper (S\&P) noise. An impulse noise detector is used, similar to [2], to identify corrupted pixels. An open-close sequence morphology algorithm is then used to first eliminate any salt noise and then the pepper noise. Finally, for detail preservation, a Block Smart Erase algorithm is proposed that will eliminate the sideeffects of implementing an open-close morphology process.

A switching median filter is used in both [6] and [10] to eliminate impulse noise. A histogram of the local window in [10] is calculated and a binary matrix created as the input to the adaptive filter. The window size of the adaptive filter is dependent on the number of corrupted pixels within a certain area of the binary matrix. Computational complexity is still an issue here as the average window size used must be kept to a minimum for processing speed.

In [8], a Spike Detection Technique (SDT) and Pixel Restoring Median Filter (PRMF) is implemented. In contrast to [10], a histogram is taken of the entire image in order to determine peaks. These peaks become the upper and lower boundary and determine the results of the corresponding binary map. The corrupted pixels are restored using a $3 \times 3$ median filter through a reiterative process.

These methods have been proven to be useful in detecting and correcting impulse noise, but have not included the additive white Gaussian noise (AWGN) that can also be seen in communication channels. Also, many of the algorithms described are too complex to be implemented on real-time systems. Our proposed method will extend what has been previously done in [8] and [10] by allowing the SDT algorithm to be more fuzzy in nature to accommodate the probability of other noise models present in an image. Various filtering techniques are then application-specific to the type of noise detected and thus, more a reliable system at the receiver.

\section{ADAPTIVE ALGORITHM METHODOLOGY}

Our proposed algorithm uses a dual-stage process of determining three metrics as follows:

- The presence of noise

- The likelihood of the noise nature (impulse, Gaussian, or uniform)

- The most effective filtering method to eliminate the noise source.
The system architecture for the proposed system is shown in Figure 1 below.

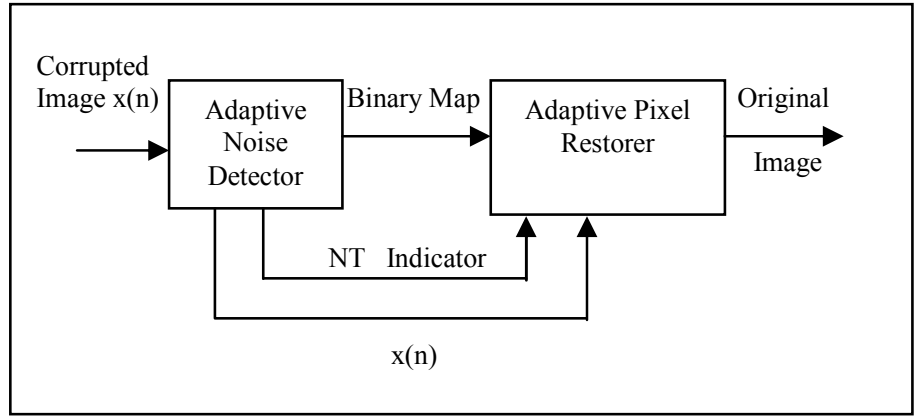

Figure 1. System Architecture

The next two subsections describe the Adaptive Noise Detector and Adaptive Pixel Restorer in more detail.

\section{A. ADAPTIVE NOISE DETECTOR}

The purpose of the Adaptive Noise Detector is to determine the type of noise, if any, exists in the current image. Similar to [8], it calculates a distance vector, D, from the image histogram array. Different boundary thresholds are then set, according to the maxima values found in $D$. Depending on where these maxima values are located determines the nature of the noise, whether it be impulse, uniform, or Gaussian.

A typical example shown in Figure 2 demonstrates the difference between edges and salt-and-pepper noise within the image. Note that the true edges have both a positive and a negative difference whereas the noisy pixels do not.
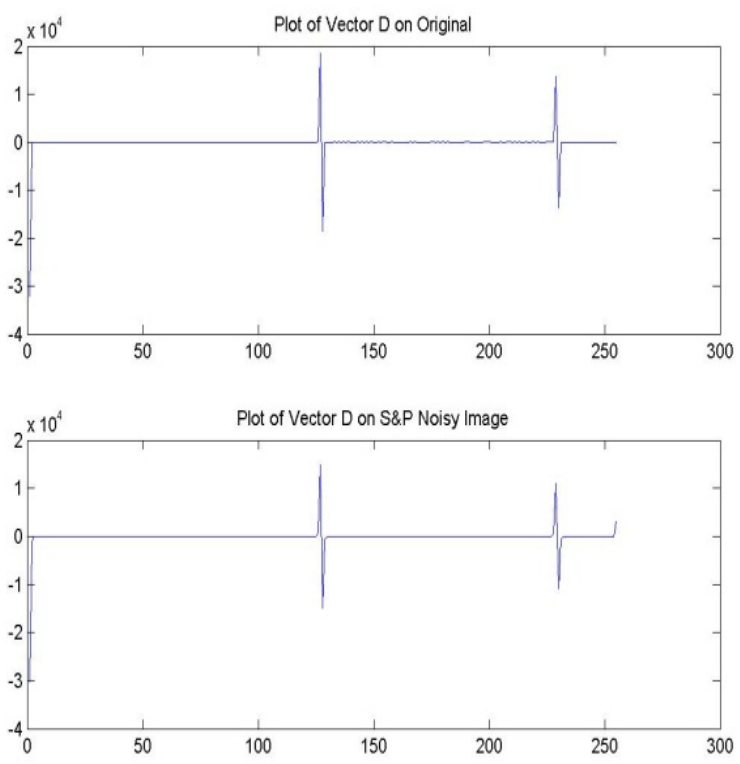

Figure 2. Plot of Difference Vector D

Once the noise has been identified, the NT Indicator is set as an input to the second stage of the system. The corrupted 
pixels are then mapped to a binary matrix with the same dimensions as the image.

\section{B. ADAPTIVE PIXEL RESTORER}

The second stage looks at the NT indicator first, which increases the processing speed. If the NT Indicator has not been set, the "corrupted image", $\mathrm{x}(\mathrm{n})$, is allowed to pass through. However, if the NT Indicator is set, then this sets one of the various noise flags.

Again, similar to the second stage of [8], the Adaptive Pixel Restorer searches through the Binary Map for pixels whose value is " 1 ". If such an instance is found and the neighborhood vector of values is not a null set, it searches through the noise flags for the ones that are set to determine what type of adaptive filtering is best suited for that instance. If the neighborhood vector of values in the vicinity of that particular pixel is null, the algorithm goes on to the next pixel value.

This process is done reiteratively until the Binary Map is cleared of 1's. The pseudo code for the Adaptive Restorer is shown below.

For an $n \times \mathrm{x}$ image, $X(n)$, with window size $3 \times 3$ :

$$
\begin{aligned}
& \text { If NT Indicator }=1 \\
& \text { Impulse Flag }=1 ; \\
& \text { Elseif NT Indicator }=2 \\
& \text { Gaussian Flag }=1 ; \\
& \text { Elseif NT Indicator }=3 \\
& \text { Uniform Flag }=1 ; \\
& \text { Else } \\
& \text { Return 0; } \\
& \text { For } i=2: 1: n-1 \\
& \text { For } j=2: 1: n-1 \\
& \text { If B }(i, j)=0 \\
& \text { Breakj loop; } \\
& \text { Else }
\end{aligned}
$$$$
\% \text { impulse noise }
$$$$
\% \text { Gaussian noise }
$$$$
\% \text { uniform noise }
$$

$$
\% \text { uncorrupted image }
$$

Check for 0's in $3 \times 3$ local binary map;

Store each pixel element in local map to vector $A$ End

$$
\begin{aligned}
& \text { If } A=\text { NULL } \\
& \text { Breakj loop; } \\
& \text { Else } \\
& \text { If Impulse Flag }==1 \\
& \quad X(i, j)=\text { Median }(A) ; \\
& \text { If Gaussian Flag==1 } \\
& \quad X(i, j)=\text { Alpha-Trim Mean }(A) ; \\
& \text { If Uniform Flag }==1 ; \\
& \quad X(i, j)=\text { Geometric_Mean }(A) ; \\
& \text { End } \\
& \text { Return } 1 ;
\end{aligned}
$$

Simulations validating the improvement in detecting various noise sources utilizing the Adaptive Noise Detector and Adaptive Pixel Restorer are demonstrated below.
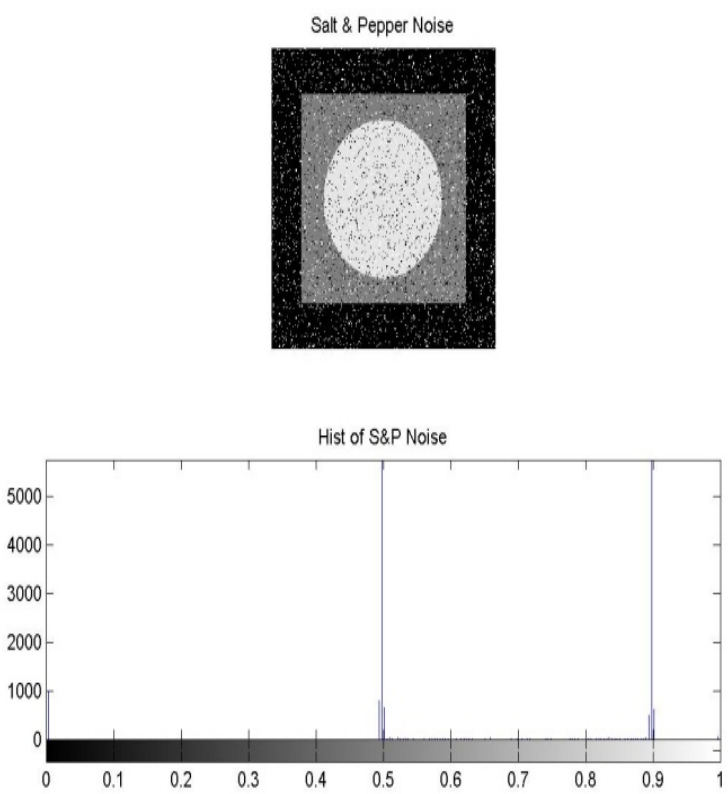

Figure 3. Histogram of S\&P Noise in Image
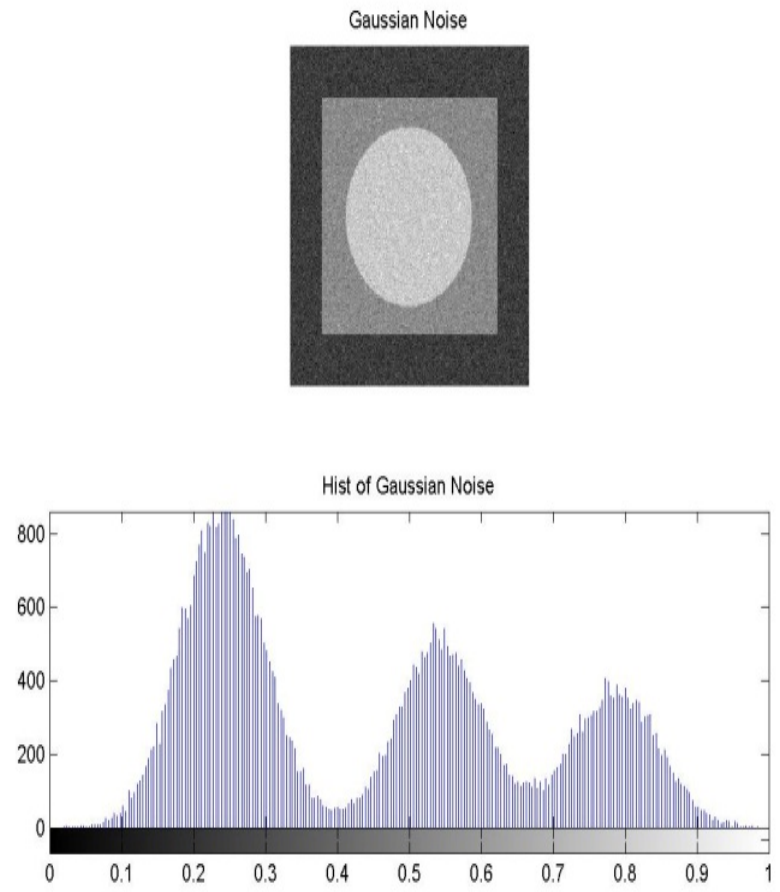

Figure 4. Histogram of Gaussian Noise in Image 
Uniform Noise
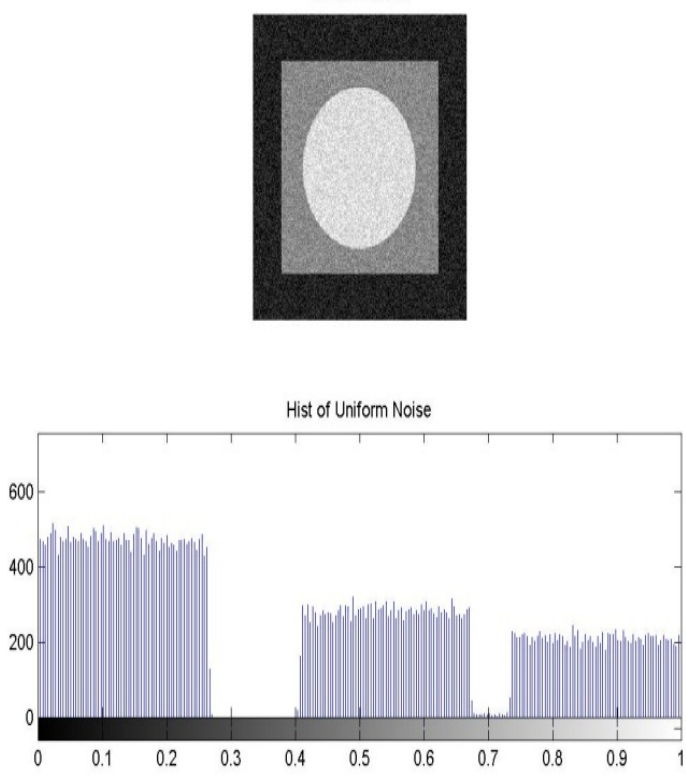

Figure 5. Histogram of Uniform Noise in Image

Figures 3, 4, and 5 show the original image corrupted by impulse, Gaussian, and uniform noises, respectively, along with their statistical features or histogram representations. The nature of each noise can easily be determined by taking the distance of neighboring histogram values and plotting this vector, as in Figure 2.

Figure 6, below, shows the reconstructed images from the second stage of the Adaptive Pixel Restorer. Comparatively, Figures 6(a), (b), and (c) are the reconstructed images from salt and pepper noise. Gaussian noise, and uniform noise, respectively.

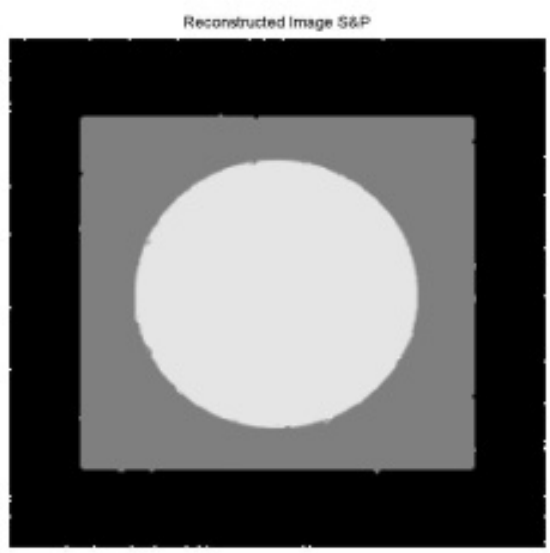

(a) Reconstructed images (S\&P)

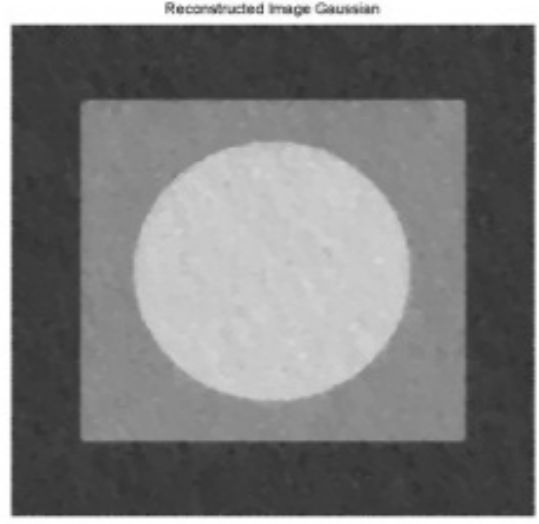

(b) Reconstructed image (Gaussian)

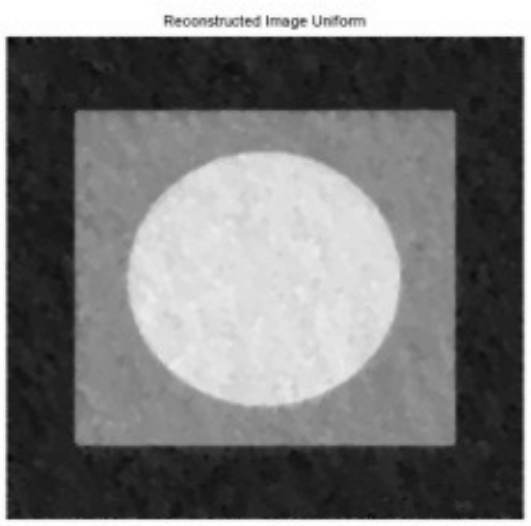

(c) Reconstructed image (uniform)

Figure 6. Reconstructed Images

Figure 7 shows the direct result of reconstructing only part of the pixel matrix, for comparison purposes.

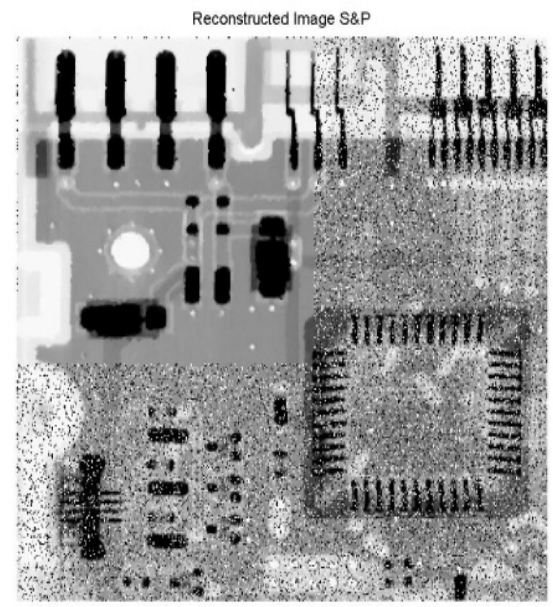

Figure 7. Partial Reconstructed S\&P Image 
Simulations were also run on images where more than one type of noise was present within the image. Figure 8 shows the presence of both additive uniform noise and salt-andpepper noise on an image. The resulting histogram shows the spikes at near 1 and 0 for the impulse noise and the additive white noise between 0.4 and 0.8 . The resulting plot of the distance vector picks up on both types of noise. The resulting partially reconstructive image can be found in Figure 9. Again, for comparison purposes, only part of the pixel matrix was processed.
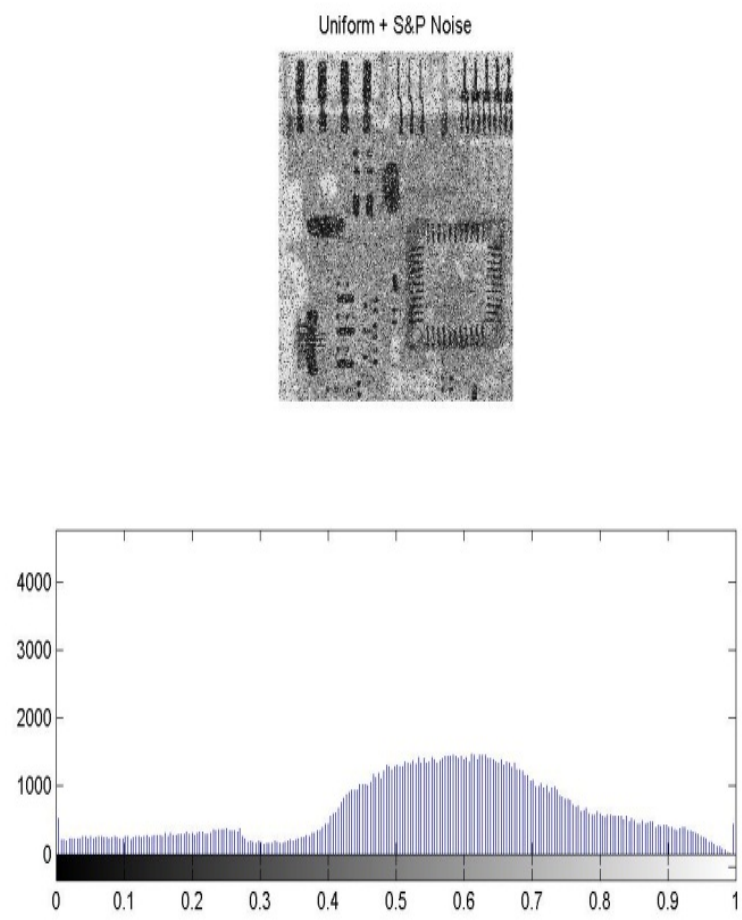

Figure 8. Image Corrupted by both Uniform and S\&P Noise

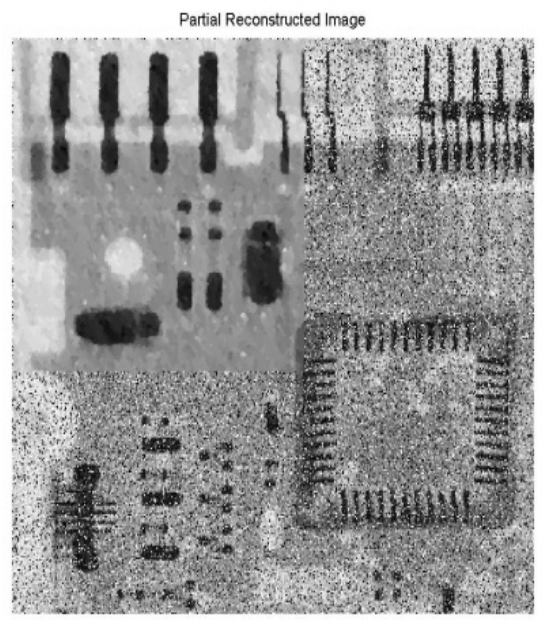

Figure 9. Partial Reconstructed Image

\section{CONCLUSION}

In this paper, an adaptive algorithm is proposed in order to dynamically determine the filtering technique needed for the nature of the noise encountered. It leads to realization of a realtime solution for multimedia applications where every nth frame is checked for noise. The simulations and experimental results showed positive and reliable detection results for a variety of noise models which is a considerably improved success rate over [8] and similar processing times for the same conditions (only impulse noise present) as [8]. This methodology can be extended into real-time applications due to the simplicity of the algorithm. However, more simulations should be done in order to verify this claim.

\section{ACKNOWLEDGMENT}

The work was supported in part by a Layman Award at University of Nebraska-Lincoln and an NSF Nebraska EPSCoR First Award.

\section{REFERENCES}

[1] S. Schulte, M. Nachtegael, V. De Witte, D. Van der Weken, E.E. Kerre, "A Fuzzy Impulse Noise Detection and Reduction Method," IEEE Transactions on Image Processing, Vol 15, Issue 5, May 2006, pp. $1153-1162$.

[2] W. Luo, "An Efficient Detail-Preserving Approach for Removing Impulse Noise in Images," In IEEE Siganl Processing Letters, Vol 13, No 7, July 2006, pp. 413-416.

[3] W. Luo, "Efficient Removal of Impulse Noise from Digital Images," IEEE Transactions on Consumer Electronics, Vol. 52, No. 2, May 2006, pp. 523-527.

[4] S. Schulte, M. Nachtegael, V. De Witte, D. Van der Weken, E.E. Kerre, "Fuzzy Two-Step Filter for Impulse Noise Reduction From Color Images," IEEE Transactions on Image Processing, Vol 15, No 11, November 2006, pp. 3568-3579.

[5] D. Ze-Feng, Y. Zhou-Ping, and X. You-Lun, "High Probability Impulse Noise-Removing Algorithm Based on Mathematical Morphology," IEEE Signal Processing Letters, Vol. 14, No. 1, January 2007, pp. 3134.

[6] T. Chen, S. Huang, C. Chen, and Z. Lin, "Adaptive Working Window for Impulse Noise Reduction," In Proceedings of the 2006 International Conference on Intelligent Information Hiding and Multimedia Signal Processing, 2006.

[7] W. Luo, and D. Dang, "An Efficient Method for the Removal of Impulse Noise," ICIP, 2006, pp. 2601-2604.

[8] S. Indu and C. Ramesh, "A Noise Fading Technique for Images Highly Corrupted with Impulse Noise," IEEE Proceedings of the International Conference on Computing: Theory and Applications, 2007.

[9] Xu, H.; Xia, X.; Guo, L.; Chen, W.; and Huang, G. "Classification-based Weighted Filter for Image Corrupted by Impulse Noise," ICSP Proceedings, 2006.

[10] Ping, W.; Junli, L.; Lu, D.; and Chen, G. "A Fast and Reliable Switching Median Filter for Highly Corrupted Images by Impulse Noise," IEEE Transactions, 2007, pp. 3427-3440.

[11] R. Gonzales, and R. Woods, Digital Image Processing, Second Edition. Prentice-Hall, Inc. 2002. 